\title{
sciendo
}

\section{Can Heart Rate Variability Biofeedback Improve Athletic Performance? A Systematic Review}

\author{
by \\ Jeffrey Cayaban Pagaduan ${ }^{1}$, Yung-Sheng Chen², James William Fell', \\ Sam Shi Xuan $W u^{3}$
}

\begin{abstract}
This systematic review was conducted to evaluate the effect of heart rate variability biofeedback (HRV BFB) on performance of athletes. Six electronic databases (Springerlink, SportDiscus, Web of Science, PROQUEST Academic Research Library, Google Scholar, and ScienceDirect) and article references were searched. Eligibility criteria were: 1. experimental studies involving athletes randomly allocated among groups (randomized control trial); 2. availability of $H R V B F B$ as a treatment compared to a control condition (CON) that involves regular sport/dance training, a placebo (PLA) or other methods of BFB; 3. performance-related variables such as a dependent index; and, 4. peer-reviewed articles written in English. Out of 660 articles, six studies were included in the systematic review which involved 187 athletes (females: $n=89$; males $n=98$ ). Six studies compared HRV BFB with a CON, three studies compared HRV $B F B$ with a PLA, and two studies differentiated HRV BFB with other methods of BFB. Findings support HRV BFB as a potential intervention to improve fine and gross motor function in athletes.
\end{abstract}

Key words: heart rate variability, biofeedback, athletes, performance, resonant frequency breathing.

\section{Introduction}

Biofeedback (BFB) as a performance enhancement strategy in athletes has been receiving notable attention among sports practitioners (Brown and Fletcher, 2016; Keilani et al., 2016; Pusenjak et al., 2015). BFB provides realtime understandable physiological information to an individual that enhances psychophysiological and affective indices (Galloway and Lane, 2005; Wilson et al., 2014). Among various approaches to $\mathrm{BFB}$ there is a breathing strategy known as heart rate variability biofeedback (HRV BFB) (Lehrer et al., 2000). HRV BFB is executed by paced breathing at a specific frequency, known as resonance frequency (RF), that elicits maximal heart rate oscillations. RF usually ranges from 4 to 6.5 breaths/min (Lehrer et al., 2000). In addition, RF exhibits a 0-degree phase shift between the heart rate and respiration as well as a 180-degree phase shift between the heart rate and blood pressure (Vaschillo et al., 2006). The physiological phenomena with HRV BFB are believed to improve autonomic function from baroreflex gain and vagal activation (Gevirtz, 2013; Lehrer et al., 2000; Lehrer and Gevirtz, 2014; Prinsloo et al., 2014; Vaschillo et al., 2006).

In an athletic setting, the first documented HRV BFB intervention was administered on wrestlers who exhibited reduced muscle relaxation disorders and an improved rate of relaxation (Vaschillo et al., 1998). A recent review conducted by Jiménez Morgan and Molina-Mora (2017) synthesized the effect of HRV BFB on athletic performance and found that $86 \%(n=6)$ of studies reviewed performance enhancement via improvement in psychophysiological variables.

\footnotetext{
1 - College of Health and Medicine, School of Health Sciences, University of Tasmania, Launceston, Tasmania, Australia.

2 - Department of Exercise and Health Sciences, University of Taipei, Taipei, Taiwan.

3 - Faculty of Health, Arts, and Design, School of Health Sciences, Department of Health and Medical Sciences, Swinburne University, Melbourne, Australia.
} 
One notable strength of the previous study was the employment of systematic procedures. On the other hand, the review included different quantitative study designs (two case reports, one quasi-experimental study, four experimental studies) which may have contributed to bias in findings. Thus, the purpose of this study was to conduct a systematic review of studies only employing randomized trials on the effect of HRV BFB on psychophysiology and exercise performance of athletes.

\section{Methods}

\section{Search Strategy}

The search was conducted between July $1^{\text {st }}$ and August $31^{\text {st }} 2017$ using the search term "heart rate variability biofeedback" and (athletes or athletic population or sport or performance or sport performance) in electronic databases: Springerlink, SportDiscus, Web of Science, PROQUEST Academic Research Library, Google Scholar, and ScienceDirect (Jiménez Morgan and Molina-Mora, 2017). In addition, a manual reference search was administered on the records found. PRISMA guidelines were used for reporting.

\section{Eligibility Criteria}

To be included in the systematic review, the studies had to meet the following criteria: 1 . experimental studies involving athletes randomly allocated among groups (randomized control trial); 2. availability of HRV BFB as a treatment compared with a control condition $(\mathrm{CON}) / \mathrm{a}$ placebo (PLA)/other BFB; 3. any performancerelated variable as a dependent index; and, 4. peer-reviewed articles written in English.

\section{Study Selection}

Literature search and selection of studies were completed by a single investigator (JP). All studies were coded and organized in an Excel spreadsheet. The second investigator (YSC) evaluated data extraction. For each article included in the systematic review, the following data were encoded: author/s and year of publication, sample size information, intervention, measured performance variable/s, and results. These studies were also assessed for 'risk of bias' using an eight-point scale from Consolidated Standards of Reporting Trials (CONSORT) statement wherein each item is answerable by 0 (absently or inadequately described) or 1 (explicitly described and present). A study with a score of 0-2 is regarded as having a high risk of bias, 3-5 with medium risk of bias, and 6-8 considered as having low risk of bias (CONSORT, 2001). A consensus was reached between JP and YSC for any disagreement presented in data extraction and CONSORT output (Table 1).

\section{Statistical Analysis}

Independent T-tests using pre-post mean differences and standard deviation of variables from HRV BFB and groups' comparison were administered. Then, corresponding Cohen's $d$ as effect size (ES) with 95\% confidence limits were calculated (Cohen, 1988; Lee, 2016; Morris, 2008). Missing pre-post mean differences and SD in studies were computed based on previous methods (Gu et al., 2015; Zu et al., 2013). ES was interpreted as small $(d=0.20)$, medium $(d=0.50)$, or large $(d=0.80)$ (Cohen, 1988). Statistical power calculation from post hoc was also conducted using $G^{*}$ Power ver 3.1 (Faul et al., 2007). Indices with alpha $=0.00$ were set at alpha $=0.01$.

\section{Results}

Figure 1 shows the flowchart and selection process of the studies. The database search indicated 656 potential articles with an additional 4 identified articles from reference lists. After removal of duplicates $(\mathrm{n}=90), 570$ articles underwent initial screening based on the article title/abstract. This process led to excluding 557 articles after failing to meet all the items in the inclusion criteria. Further assessment for eligibility of 13 full articles led to removal of seven studies leaving six articles included in the analysis.

Participants

The six studies included a total of 187 (females: $\mathrm{n}=89$; males $\mathrm{n}=98$ ) athletes including: 60 (females: $\mathrm{n}=27$; males: $\mathrm{n}=33$ ) university, state, and national basketball athletes; 24 (females: $\mathrm{n}=$ 12; males: $\mathrm{n}=12$ ) university, state, and national standard long distance runners; 20 male professional soccer players; and, 84 (females: $\mathrm{n}=$ 51; males: $\mathrm{n}=33$ ) university student dancers.

Experimental protocols

Of the six studies included, all studies compared HRV BFB and a control condition $(\mathrm{CON})$ wherein a $\mathrm{CON}$ involved regular dance/sport training. Three studies also compared 
HRV BFB and a placebo (PLA) with two studies using motivational video (Paul and Garg, 2012; Paul et al., 2012) and one using choreology (Gruzelier et al., 2014). Two studies compared HRV BFB with the alternative BFB intervention neurofeedback (NFB) which utilized electronenchaphalogram (EEG) signals (Gruzelier et al., 2014; Raymond et al., 2005).

Interventions

The studies applied a variety of HRV BFB protocols. Two studies utilized 10 consecutive days of HRV BFB administered at 20-min/day (Paul and Garg, 2012; Paul et al., 2012). Other studies followed protocols for HRV BFB suggested by Lehrer et al. (2000) using 10 formal sessions with two 20-min daily sessions at convenience (Choudhary, et al., 2016; Gruzelier et al., 2014; Raymond et al., 2005). Two studies following a protocol set by Lehrer et al. (2000) lasted for 10 weeks, while the study of Raymond et al. (2005) was carried out for 4 weeks.

Rusciano et al. (2017) administered HRV BFB for fifteen 30-min sessions (twice a week). The first three sessions followed the protocol set by Lehrer et al. (2000). Then sessions $4-9$ combined HRV BFB with other biofeedback schemes including a skin conductance level (SCL), electromyography (EMG) of masseter and a posterior cervical region and hand temperature. The remaining sessions $\left(10^{\text {th }}-15^{\text {th }}\right)$ integrated HRV BFB with math tasks, hyperventilation, and winning and losing video games.

Instruments

Different HRV BFB equipment was utilized for HRV BFB. Two studies utilized freezeframer from Boulder Creek, California, US (Gruzelier et al., 2015; Raymond et al., 2005). Three studies used Biograph Pro Comp Infinity 5.0 from Thought Technology Ltd., Canada (Choudhary et al., 2016; Paul and Garg, 2012; Paul et al., 2012). Rusciano et al. (2017) used HRV BFB with Nexus 10 Mark II hardware and Biotrace1 commercial software from Mind Media, Herten, Netherlands.

\section{Performance Evaluation}

Studies included in the review assessed various performance related variables. Changes in performance (\%) and effect sizes with 95\% confidence limits of HRV BFB and groups' comparison, as well as study power are displayed in Table 2.
Choudhary et al. (2016) determined 5-km performance of track athletes. The study of Paul et al. (2012) assessed performance using movement and choice reaction time (RT) along with a 3-min shooting score. Paul and Garg (2012) measured weaving in and around cones whilst dribbling for $30 \mathrm{~s}, 30$-s passing with specified targets on the wall, and 3-min shooting at marked perimeters. Rusciano et al. (2017) established visual tracking RT of professional soccer players with and without a target. Accuracy under congruent and incongruent stimuli using a Stroop task was also determined. Further, injury prevention was identified from attendance records (days present and absent or differential training days - the number of days on which athletes followed personalized training due to recent injuries). In the study of Gruzelier et al. (2014), four dance experts rated dance performance for artistry and technique. Raymond et al. (2005) utilised two qualified dance assessors that evaluated dance performance considering a technicality, musicality, timing, partnering skill, performing flair, and overall execution from a customized scale with scoring of one to five. In addition, rating scores were divided by the number of practice sessions for each dancer to derive "improvement per practice session". Then, scores were averaged to get the group score from "practice-corrected average difference". Characteristics of each study are presented in Table 3.

Performance Outcomes

HRV BFB vs. CON

For gross motor skill executed in relatively short duration comparing HRV BFB and a a CON, Paul and Garg (2012) demonstrated non-significant difference in dribbling between the HRV BFB and the CON group at $p=0.06$, $\mathrm{ES}[95 \% \mathrm{CL}]=0.89[0.60,1.18]$. On the other hand, the HRV BFB group presented significant enhancement in passing and shooting at $p<0.001$, $2.14[1.79,2.49]$ and $p<0.001,2.00[1.66,2.34]$, respectively. In a similar study, Paul et al. (2012) found significant improvement in 3-min shooting after HRV BFB compared to a CON at $p=0.01$, $1.38[1.07,2.36]$. Raymond et al. (2005) observed non-significant difference in performance scores of dancers at $p=0.40,0.55[-0.06,1.77]$. No significant difference in practice-corrected difference scores at $p=0.31,0.66[0.04,1.89]$ was also found. 


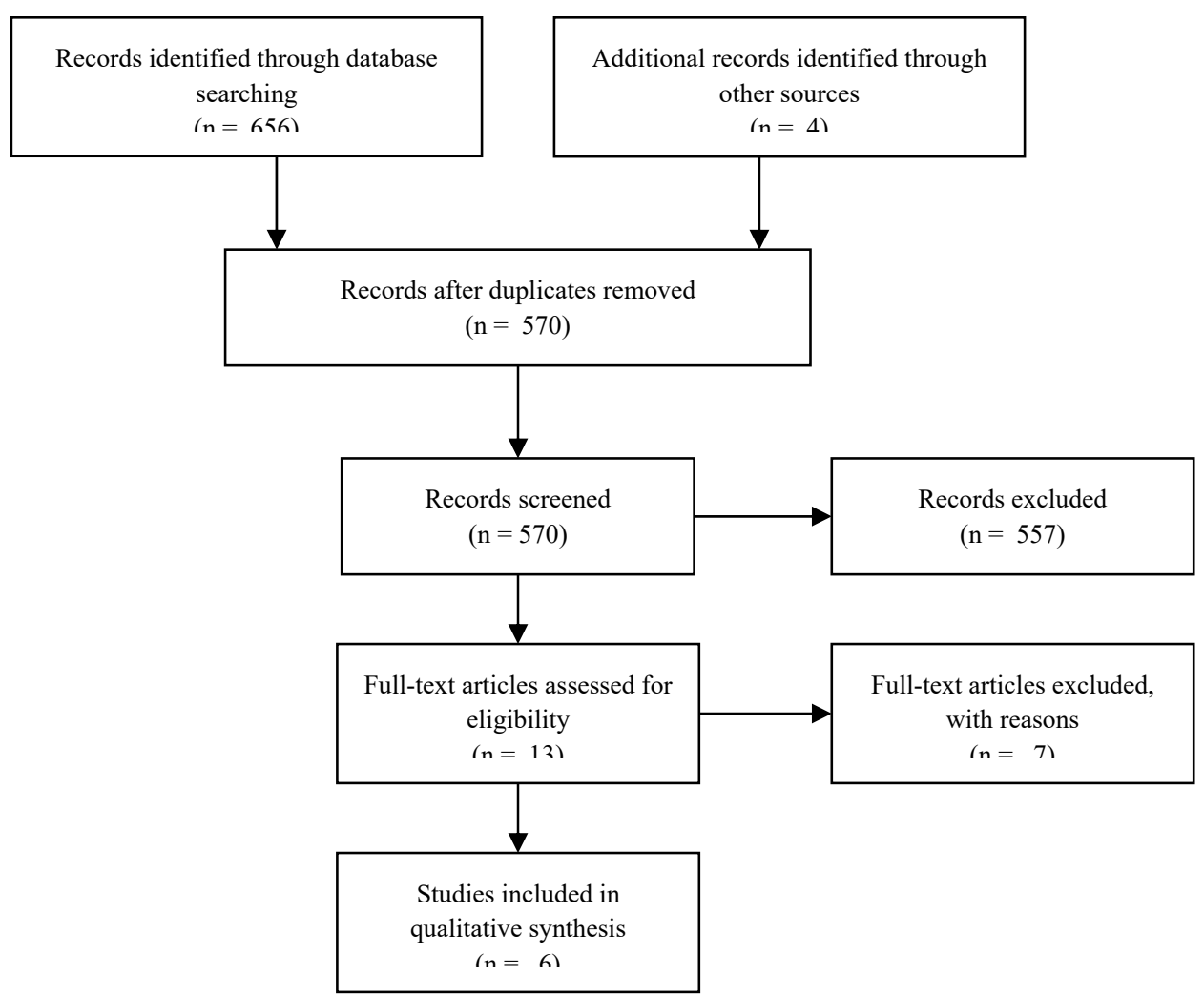

Figure 1

Flow diagram of the search process

Table 1

CONSORT scores of articles included for systematic review

\begin{tabular}{|c|c|c|c|c|c|c|c|c|c|}
\hline References & Item 1 & Item 2 & Item 3 & Item 4 & Item 5 & Item 6 & Item 7 & Item 8 & Total \\
\hline $\begin{array}{c}\text { Choudhary et al. } \\
\text { (2016) }\end{array}$ & 1 & 0 & 0 & 0 & 0 & 1 & 1 & 0 & 3 \\
\hline $\begin{array}{c}\text { Gruzelier et al. } \\
\text { (2014) }\end{array}$ & 0 & 0 & 1 & 0 & 0 & 1 & 1 & 0 & 3 \\
\hline $\begin{array}{l}\text { Paul and Garg } \\
\text { (2012) }\end{array}$ & 1 & 0 & 1 & 0 & 1 & 1 & 1 & 0 & 5 \\
\hline $\begin{array}{l}\text { Paul et al. } \\
\text { (2012) }\end{array}$ & 1 & 0 & 1 & 0 & 1 & 1 & 1 & 0 & 5 \\
\hline $\begin{array}{c}\text { Raymond et al. } \\
\text { (2005) }\end{array}$ & 1 & 0 & 1 & 0 & 0 & 1 & 1 & 0 & 4 \\
\hline $\begin{array}{l}\text { Rusciano et al. } \\
\text { (2017) }\end{array}$ & 1 & 0 & 1 & 0 & 0 & 1 & 1 & 1 & 5 \\
\hline
\end{tabular}

Item 1 - Were the groups comparable at baseline on key characteristics? Item 2 -Did the study include a true control group (randomised participants - not a comparison group)? Item 3 - Was the randomisation procedure adequately described and carried out? Item 4 -Did the study report a power calculation and was the study adequately powered to detect intervention effects? Item 5 - Were the assessors blinded to treatment allocation at baseline and posttest? Item 6 - Did at least $80 \%$ of the participants complete follow-up assessments? Item 7 -Did the study analyses account for potential differences at baseline? Item 8 - Did the study compute effect sizes? 
Table 2

Effect Size with 95\% Confidence Interval of Studies in HRV BFB vs Comparison Group

\begin{tabular}{|c|c|c|c|c|c|c|c|c|}
\hline \multirow{2}{*}{$\frac{\text { Study }}{\text { HRV BFB vs CON }}$} & \multirow[t]{2}{*}{ Parameter } & \multicolumn{2}{|c|}{$\%$ Change } & \multirow{2}{*}{$\frac{\mathrm{p} \text { - }}{\text { value }}$} & \multirow[t]{2}{*}{ ES } & \multicolumn{2}{|c|}{$\begin{array}{c}95 \% \text { Confidence } \\
\text { Interval } \\
\end{array}$} & \multirow[t]{2}{*}{ Power } \\
\hline & & HRV BFB & $\mathrm{CON}$ & & & LL & UL & \\
\hline Choudhary et al. (2016) & 5-km running & -13.0 & -0.71 & 0.16 & -0.60 & -0.84 & 0.22 & 0.51 \\
\hline \multirow[t]{3}{*}{ Paul and Garg (2012) } & dribbling & 25.1 & 6.54 & 0.06 & 0.89 & 0.60 & 1.18 & 0.50 \\
\hline & passing & 76.6 & 13.2 & 0.00 & 2.14 & 1.79 & 2.49 & 0.96 \\
\hline & shooting & 104 & 9.43 & 0.00 & 2.00 & 1.66 & 2.34 & 0.93 \\
\hline \multirow[t]{3}{*}{ Paul et al. (2012) } & $\begin{array}{l}\text { shooting } \\
\text { movement }\end{array}$ & 98.1 & 27.3 & 0.01 & 1.38 & 1.07 & 2.36 & 0.59 \\
\hline & time & -17.1 & 0.00 & 0.05 & -0.95 & -1.24 & -0.02 & 0.52 \\
\hline & choice RT & -21.3 & 0.00 & 0.01 & -1.23 & -1.53 & -0.27 & 0.47 \\
\hline Raymond et al. (2005) & $\begin{array}{c}\text { dance } \\
\text { performance } \\
\text { practice- } \\
\text { corrected } \\
\text { difference }\end{array}$ & 15.1 & NA & 0.31 & 0.66 & 0.04 & 1.89 & 0.53 \\
\hline \multirow[t]{7}{*}{ Rusciano et al. (2017) } & $\begin{array}{c}\text { target absent } \\
\text { RT } \\
\text { target present }\end{array}$ & -29.4 & -1.58 & 0.00 & -1.72 & -2.05 & -0.70 & 0.82 \\
\hline & $\begin{array}{c}\mathrm{RT} \\
\text { congruent task }\end{array}$ & -22.6 & -11.0 & 0.31 & -0.47 & -0.72 & 0.42 & 0.53 \\
\hline & $\begin{array}{c}\text { accuracy } \\
\text { incongruent }\end{array}$ & 10.5 & 0.57 & 0.00 & 2.64 & 2.26 & 3.84 & 1.00 \\
\hline & task accuracy & 15.6 & 0.95 & 0.00 & 2.94 & 2.54 & 4.20 & 1.00 \\
\hline & days present & NA & NA & 0.00 & 1.51 & 1.20 & 2.50 & 0.69 \\
\hline & $\begin{array}{l}\text { days absent } \\
\text { differential }\end{array}$ & NA & NA & 0.00 & -1.52 & -1.83 & -0.52 & 0.69 \\
\hline & training & NA & NA & 0.18 & -0.62 & -0.90 & 0.28 & 0.51 \\
\hline HRV BFB vs PLA & & HRV BFB & PLA & & & & & \\
\hline \multirow[t]{3}{*}{ Paul and Garg (2012) } & dribbling & 25.1 & 3.06 & 0.04 & 0.99 & 0.70 & 1.92 & 0.51 \\
\hline & passing & 76.6 & 23.9 & 0.01 & 1.38 & 1.07 & 2.36 & 0.59 \\
\hline & shooting & 104 & 28.8 & 0.00 & 1.63 & 1.31 & 2.64 & 0.77 \\
\hline \multirow[t]{3}{*}{ Paul et al. (2012) } & $\begin{array}{l}\text { shooting } \\
\text { movement }\end{array}$ & 98.1 & 30.8 & 0.00 & 1.39 & 1.08 & 2.37 & 0.60 \\
\hline & time & -17.1 & 0.00 & 0.42 & -0.37 & -0.64 & 0.52 & 0.56 \\
\hline & choice RT & -21.3 & 0.00 & 0.00 & -1.51 & -1.82 & -0.51 & 0.69 \\
\hline HRV BFB vs NFB & dance & HRV BFB & NFB & & & & & \\
\hline Raymond et al. (2005) & $\begin{array}{l}\text { performance } \\
\text { practice- } \\
\text { corrected } \\
\text { difference }\end{array}$ & 15.1 & 12.2 & 0.86 & 0.12 & -0.51 & 1.39 & 0.86 \\
\hline
\end{tabular}

ES - effect size; LL - lower limit; UL - upper limit; $N A$ - not applicable 
Table 3a

HRV BFB and Athlete Performance

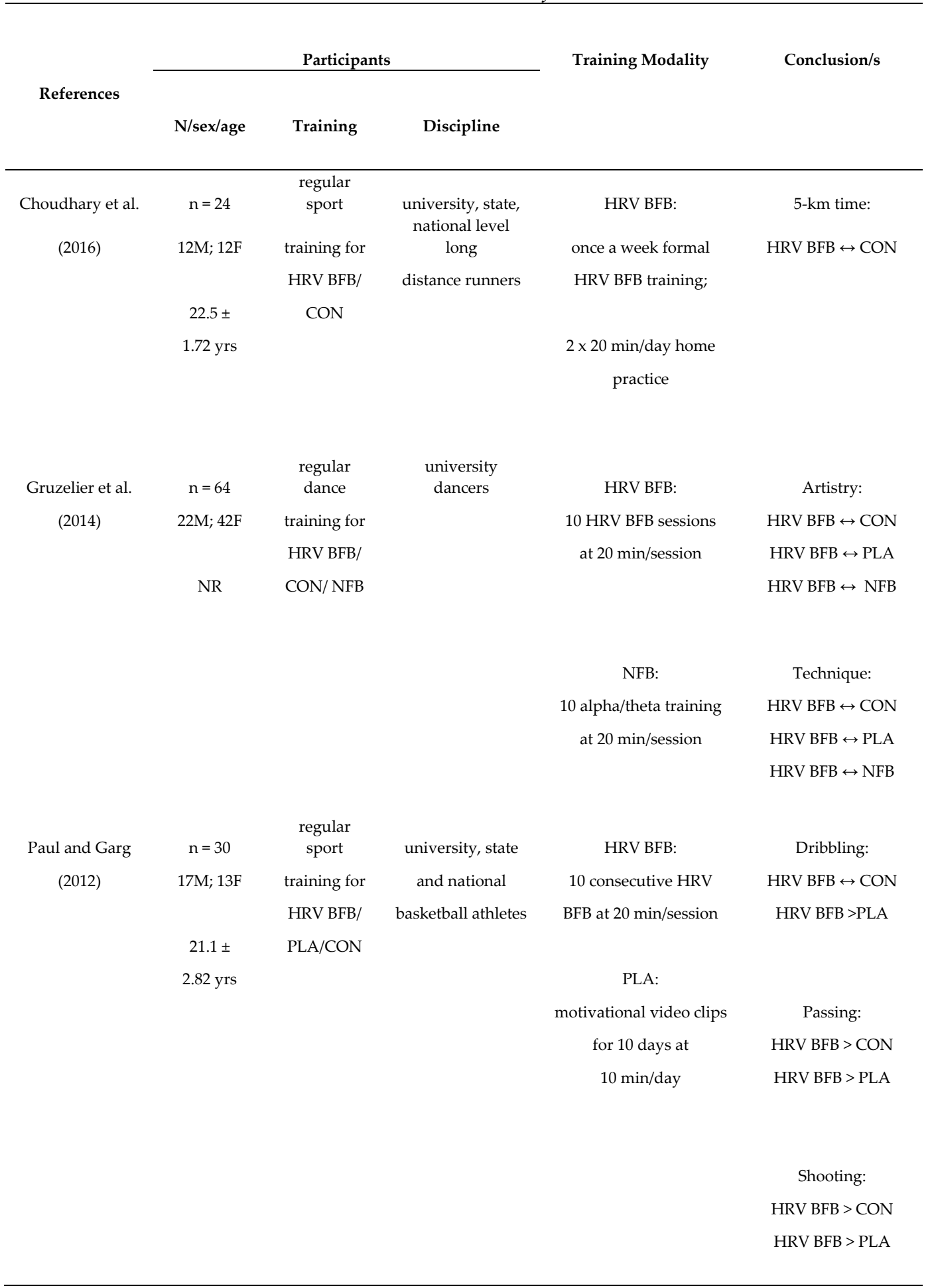

HRV BFB - heart rate variability biofeedback; PLA - placebo; CON - control; NFB - neurofeedback; NR - not reported; SCL - skin conductance level;

EMG - electromyography; $\boldsymbol{R T}$ - reaction time 


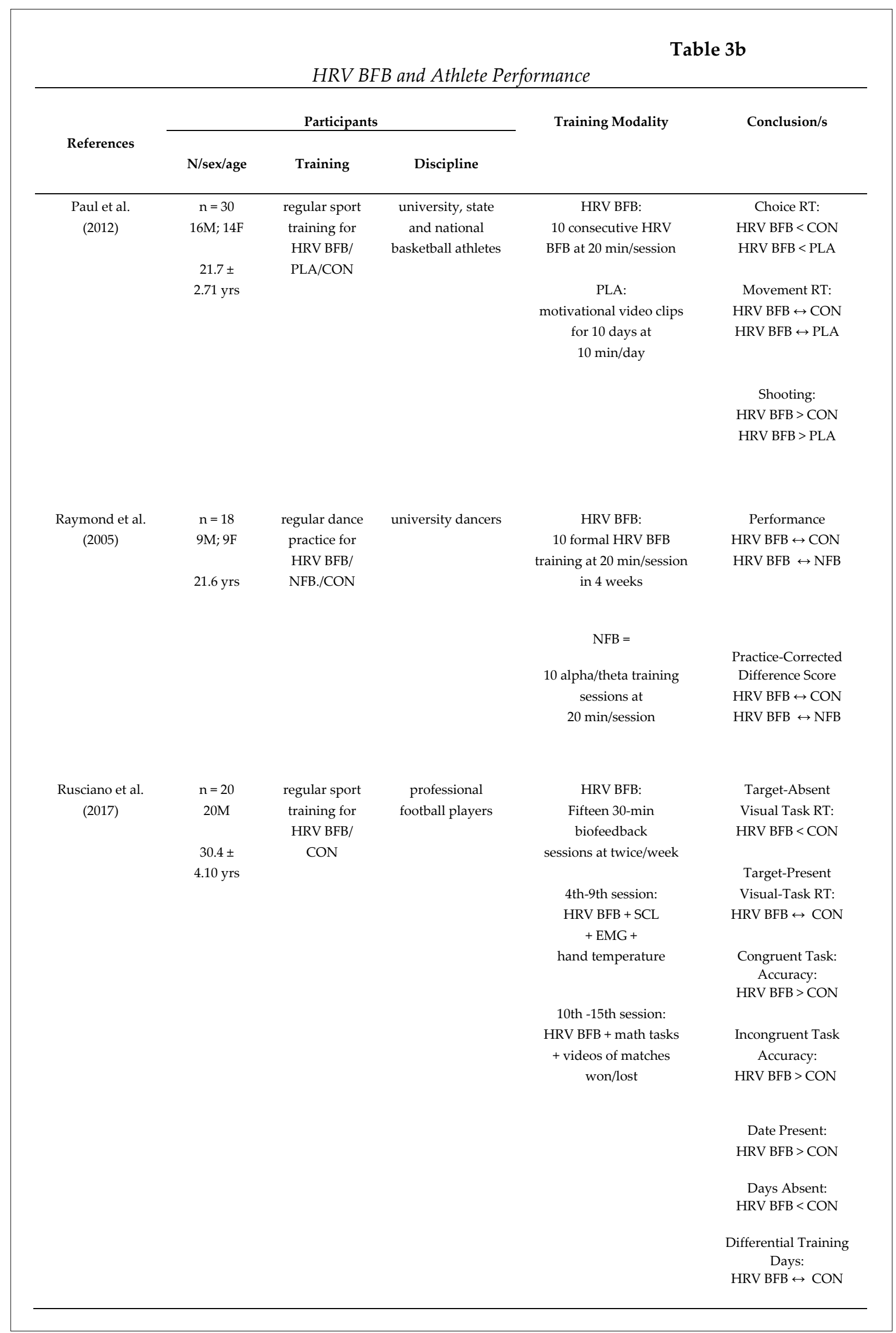


Gruzelier et al. (2014) found that university dancers exhibited non-significant outcomes in artistry and technique between HRV $\mathrm{BFB}$ and CON groups.

In exercise of longer duration, Choudhary et al. (2016) reported no significant improvement in gross motor skill movement via $5-\mathrm{km}$ performance between the HRV BFB group and a $\mathrm{CON}$ at $p=0.16$, ES[95\% CL] $=-0.60[-0.84,0.22]$.

In fine motor ability, Paul et al. (2012) found no significant reduction in movement time seen after HRV BFB and a CON at $p=0.05,-0.95[-$ $1.24,-0.02]$. On the other hand, there was significantly shorter choice reaction time found after HRV BFB compared to a CON at $p=0.01$, 1.23[-1.53, -0.27]. Rusciano et al. (2017) detected significant improvement in visual tracking scores with a target absent stimulus among soccer athletes after HRV BFB compared to a CON at $p=$ $0.00,-1.72[-2.05,-0.70]$. However, visual tracking with a target present stimulus did not significantly improve after HRV BFB compared to a $\mathrm{CON}$ at $p=0.31$; ES $=-0.47[-0.75,0.42]$. Furthermore, soccer players after HRV BFB significantly increased accuracy under congruent stimuli at $p=0.00,2.64[2.26,3.84]$ compared to a CON. Similarly, the HRV BFB group showed significantly higher accuracy following incongruent stimuli compared to a $\mathrm{CON}$ at $p=$ $0.00,2.94[2.54,4.20]$.

Rusciano et al. (2017) found significantly reduced absence from sport training sessions compared with a CON at $p=0.00,1.51[1.20,2.50]$. The number of absences from training in the entire sport season that followed treatment from injury was also significantly lower in the HRV BFB group than a CON at $p=0.00,-1.52[-1.83$, 0.52]. Differential training between the HRV BFB group and a CON was not significantly different, $p=0.18,-0.62[-0.90,0.28]$.

HRV BFB vs. PLA

Paul and Garg (2012) discovered a significantly higher dribbling score in the HRV BFB group than a PLA at $p=0.04,0.99[0.70,1.92]$. Passing also significantly improved in the HRV BFB group compared with a PLA at $p=0.01$, $1.38[1.07,2.36]$. The HRV BFB group significantly increased shooting performance compared to a PLA, $p=0.00,1.63[1.31,2.64]$. In the study of Paul et al. (2012), athletes after HRV BFB significantly increased shooting scores at $p=0.01,1.39[1.08$,
2.37]. In dance, artistry and technique were similar in both the HRV BFB and the PLA group (Gruzelier et al., 2014).

For fine motor task ability examining HRV BFB and a PLA, non-significant improvement in movement time was identified between the HRV BFB group and a PLA at $p=$ $0.42,-0.37[-0.64,0.52]$. However, the HRV BFB group presented significantly lower choice reaction time than the PLA at $p=0.00,-1.51[-1.82$, 0.51].

\section{$H R V B F B$ vs. other BFB intervention}

Raymond et al. (2005) presented similar both performance and practice-corrected performance after HRV BFB and NFB at $p=0.86$, $0.12[-0.51,1.31]$ and $p=0.94,0.05[-0.58,1.31]$, respectively. Gruzelier et al. (2014) recorded nonsignificant difference in artistry and technique between HRV BFB and NFB.

\section{Discussion}

The purpose of this systematic review was to examine the effect of HRV BFB on performance indices of athletes. When compared to a control condition $(\mathrm{CON})$ that involved regular training only, HRV BFB displayed contrasting effects on the gross motor skills during short duration performance. In addition, the effect of HRV BFB on gross motor function during exercise of longer duration is unclear. Confounding results in fine motor skills after HRV BFB were also identified in comparison with a CON.

In this study, short duration $\quad<10$ minutes) gross motor tasks after HRV BFB delivered conflicting results. For example, Paul and Garg (2012) showed improved shooting and passing performance in basketball players after HRV BFB compared to a CON. However, nonsignificant difference in dribbling was seen after HRV BFB and a CON. In a similar study, Paul et al. (2012) recorded increased shooting following HRV BFB compared to a CON. Raymond et al. (2015) presented no significant difference in dance performance and practice-corrected difference in HRV BFB and a CON. The conflicting results in short duration gross motor ability can be attributed to underpowered trials (Table 3). Future research in HRV BFB and gross motor abilities should employ adequate sample size to facilitate enough power to detect meaningful difference (Biau et al., 2008). 
The influence of HRV BFB on longer duration (> $10 \mathrm{~min}$ ) gross motor performance in comparison with a $\mathrm{CON}$ is unclear due to limited literature. In the study of Choudhary et al. (2016), $5-\mathrm{km}$ performance of athletes in the HRV BFB and the CON group was not significantly different. However, a notable trend in running enhancement of $13.0 \% \quad(E S=-0.60)$ was demonstrated by athletes from the HRV BFB group. On the other hand, the CON group displayed a $0.71 \%$ change in performance. Better performance exhibited by athletes following HRV BFB suggests the positive influence of HRV BFB on longer duration gross motor ability. HRV BFB may have reduced physiological stress of athletes from increased blood flow to internal organs, elevated minimum left ventricular elastance, baroreflex sensitivity gain, and improved pulmonary function (Fonoberova et al., 2014; Lehrer et al., 2003; McEwen and Seewan, 2003). More studies are needed to establish the effect of HRV BFB on gross motor ability.

In another light, the fine motor ability of athletes after HRV BFB and a CON displayed conflicting results. Paul et al. (2012) observed improved choice reaction time after HRV BFB. Conversely, no significant enhancement in movement reaction time existed between the HRV BFB and the CON group. Rusciano et al. (2017) found enhancement in reaction time with a target absent stimulus after HRV BFB. However, there was no significant change in the target present stimulus in both groups. Non-significant findings in fine motor ability between HRV BFB and CON can also be due to insufficient sample size leading to low statistical power. In the study of Paul et al. (2012), employment of unequal sample size of males and females between groups may have influenced the non-significant results (Rusticus and Lovato, 2014). Males and females utilize different processing strategies in reaction time wherein males demonstrate faster reaction time than females (Adam et al., 1999; Dane and Ezurumluoglu, 2003). This can be supported by the greater vagal activity observed in females due to the presence of more oestrogen than in males (Dart et al., 2002; Du et al., 2006; Koenig and Thayer, 2016). Oestrogen improves the activity of choline uptake and synthesis of acetylcholine, thereby increasing vagal function (Dart et al., 2002). In addition to unequal distribution of males and females between groups, inclusion of athletes from various competitive levels in the study by Paul et al. (2012) may have increased the variability in fine motor skill execution (Carillo et al., 2011; Mückschel et al., 2014). Indeed, previous studies have shown better reaction times in elite athletes compared to non-elite athletes (Loureiro Jr and Freitas, 2012; van de Water et al., 2017; Verbugh et al., 2016). Addressing the aforementioned shortcomings when examining fine motor skill with HRV BFB training in future studies should be warranted.

Although inconsistent results were observed in HRV BFB and a CON, there is a favourable trend in performance enhancement towards HRV BFB in overall motor function. Possible physiological mechanisms of HRV BFB can be explained by the neurovisceral integration model (Porges, 2009; Thayer and Lane, 2009). HRV BFB increases activation of the vagal nerve (Gevirtz, 2013; Lehrer and Gevirtz, 2014). The vagal nerve is connected to the anterior cingulate cortex, the brain region that plays a crucial role for multi-component behaviour (Duncan, 2010; Mayer, 2011; Mückschel et al., 2014). HRV BFB may have facilitated the production of neurotransmitters responsible for improving fine and gross motor function (Beste et al., 2016; Hassert et al., 2004; Juster et al., 2010; Sellaro et al., 2015; Steenbergen et al., 2015; Yildiz et al., 2014). However, the exact mechanism affecting performance from improvement in vagal function is unknown. Future studies should be sufficiently powered, and include biochemical markers to elucidate the mechanistic properties of HRV BFB.

An interesting finding in this review is the increased attendance of athletes in training with HRV BFB. Rusciano et al. (2017) recorded an $\sim 86 \%$ presence in training and $\sim 4 \%$ absent rate out of 240 training sessions in soccer players in HRV $\mathrm{BFB}$ training. For athletes from a $\mathrm{CON}$ group, the rates of attendance and absence were $\sim 73 \%$ and $\sim 14 \%$, respectively. Although differential training was not significantly different between groups, athletes following HRV BFB presented lower differential training of $\sim 9 \%$ compared to a CON $(\sim 13 \%)$. The increased attendance, reduced absences, and a lower trend in differential training among athletes under HRV BFB may be related to improved physiological adaptations leading to resilience to stressors (Ivarsson and Johnson, 2010; 
Porges, 2007, 2009; Thayer and Lane, 2009). Thus, HRV BFB can also be a promising intervention in increasing athlete's attendance in training by reduction of the risk of injuries.

Aside from comparing HRV BFB and a $\mathrm{CON}$, the researchers also found improvement in short-duration gross motor performance with HRV BFB compared to a PLA. Paul and Garg (2012) displayed increased dribbling, shooting, and passing after HRV BFB compared to a PLA. Similarly, Paul and Garg (2012) showed improved shooting after HRV BFB compared to a PLA. Implications from these results point to HRV BFB as a superior alternative compared with a PLA in improving gross motor function of short duration.

The influence of HRV BFB on fine motor skill differentiated with a PLA is vague due to the scarcity of literature. Paul et al. (2012) recorded non-significant difference in movement time between HRV BFB and a PLA. On the other hand, HRV BFB presented enhanced choice reaction time in HRV BFB compared to a CON. Therefore, additional HRV BFB studies including a PLA are needed.

It is necessary to acknowledge that the outcomes of this study are limited to the type of athletes included in the analyses. Generalization of results should be avoided. In addition, this review evaluated performance indices to provide practitioners with a simple and direct link on HRV BFB and performance.

\section{Conclusion}

Findings of this study indicate the potential ergogenic ability of HRV BFB in improving fine and gross motor skills of athletes.

\section{References}

Adam JJ, Paas EG, Buekers MJ, Wuyts IJ, Spijkers WA, Wallmeyer P. Gender differences in choice reaction time: evidence for differential strategies. Ergonomics, 1999; 42(2): 326-335

Altman DG, Schulz KF, Moher D, Egger M, Davidoof F, Gøtzsche PC, Lang, T; CONSORT GROUP (Consolidated Standards of Reporting Trials). Ann Intern Med, 2001; 134(8): 663-694

Beste C, Steenbergen L, Sellaro R, Grigoriadou S, Zhang R, Chmielewski W, Stock AK Colzato L. Effects of concomitant stimulation of the GABAergic and norepinephrine system on inhibitory control - a study using transcutaneous vagus nerve stimulation. Brain Stimul, 2016; 9(6): 811-818

Biau DJ, Kernéis S, Porcher R. Statistics in brief: the importance of sample size in the planning and interpretation of medical research. Clin Orthop Relat Res, 2008. 466(9): 2282-2288

Brown DJ, Fletcher D. Effects of psychological and psychosocial interventions on sport performance: a metaanalysis. Sports Med, 2016; 47(1): 77-99

Carrillo AE, Christodoulou VX, Koutedakis Y, Flouris, AD. Autonomic nervous system modulation during an archery competition in novice and experienced adolescent archers. J Sports Sci, 2011; 29(9): 913-917

Choudhary R, Triveti V, Choudhary SG. Effect of heart rate variability biofeedback training on the performance of track athletes. IJTRR, 2016; 5(4): 116-174

Cohen J. Statistical power analysis for the behavioural sciences ( $2^{\text {nd }}$ ed). Hillsdale, NJ: Erlbaum, 75-144; 1988

Dane S, Erzurumluoglu A. Sex and handedness differences in eye-hand visual reaction times in handball players. Int J Neurosci, 2003; 13(7): 923-929

Dart AM, Du XJ, Kingwell BA. Gender, sex hormones and autonomic nervous control of the cardiovascular system. Cardiovasc Res, 2002; 53: 678-687

Du XJ, Fang L, Kiriazis H. Sex dimorphism in cardiac pathophysiology: experimental findings, hormonal mechanisms, and molecular mechanisms. Pharmacol Ther, 2006; 111: 434-475

Duncan J. The multiple-demand (MD) system of the primate brain: mental programs for intelligent behaviour. Trends Cogn Sci, 2014; 14(4): 172-179

Faul F, Erdfelder E, Lang AG, Buchner A. G*Power 3: a flexible statistical power analysis program for the social, behavioural, and biomedical sciences. Behav Res Methods, 2007; 39(2): 175-191

Fonoberova M, Mezić I, Buckman JF, Fonoberov V, Mezić A, Vaschillo EG, Mun EY, Vaschillo B, Bates ME. A computational physiology approach to personalized treatment models: the beneficial effects of slow breathing on the human cardiovascular system. Am J Physiol Heart Circ Physiol, 2014; 307(7): 1073-1091 
Galloway S, Lane A. The effects of biofeedback training on elite junior tennis players. J Sports Sci, 2005; 23(1112), 1247

Gevirtz R. The promise of heart rate variability biofeedback: evidence-based applications. Biofeedback, 2013; 4: $110-120$

Gruzelier JH, Thompson T, Redding E, Brandt R, Steffert T. Application of alpha/theta neurofeedback and heart rate variability training to young contemporary dancers: state anxiety and creativity. Int $J$ Psychophysiol, 2014; 93: 105-111

Gu S, Shi J, Tang Z, Sawhney M, Hu H, Shi L, Fonseca V, Dong H. Comparison of glucose lowering effect of metformin and acarbose in type 2 diabetes mellitus: a meta-analysis. PLoS One, 2015; 10(5): e0126704

Hassert DL, Miyashita T, Williams CL. The effects of peripheral vagal nerve stimulation at a memorymodulating intensity on norepinephrine output in the basolateral amygdala. Behav Neurosci, 2004; 118(1): 79-88

Ivarsson A, Johnson U. Psychological factors as predictors of injuries among senior soccer players. a prospective study. J Sports Sci Med, 2010; 9(2): 347-352

Jiménez Morgan S, Molina Mora SJ, Effect of heart rate variability biofeedback on sports performance. Appl Psychophysiol Biofeedback, 2017; 42(3): 235-245

Juster RP, McEwen BS, Lupien SJ. Allostatic load biomarkers of chronic stress and impact on health and cognition. Neuro Sci Biobehav Rev, 2010; 35(1): 2-16

Keilani M, Hasenöhrl T, Gartner I, Krall C, Fürnhammer J, Cenik F, Crevenna R. Use of mental techniques for competition and recovery in professional athletes. Wien Klin Wochenschr, 2016; 128: 315-319

Koenig J, Thayer JF. Sex differences in healthy human heart rate variability: a meta-analysis. Neurosci Behav Rev, 2016; 64: 288-310

Lee DK. Alternatives to $p$ value: confidence interval and effect size. Korean J Anesthesiol, 2016; 69(6): 555-562

Lehrer PM, Gevirtz R. Heart rate variability biofeedback: how and why does it work? Front Psychol, 2014; 5: 756

Lehrer PM, Vaschillo E. Vaschillo B. Resonant frequency biofeedback training to increase cardiac variability: rationale and manual for training. Appl Psychophysiol Biofeedback, 2000; 25(3): 177-191

Lehrer PM, Vaschillo E, Vaschillo B, Lu SE, Eckberg DL, Edelberg R, Shih WJ, Lin Y, Kuusela TA, Tahvanainen KU, Hamer RM. Heart rate variability biofeedback increases baroreflex gain and peak expiratory flow. Psychosom Med, 2003; 65(5): 796-805

Loureiro Jr LdFB, Freitas PBd. Influence of the performance level in badminton players in neuromotor aspects during a target-pointing task. Rev Bras Med Esporte, 2012; 18(3): 203-207

Mayer EA. Gut feelings: the emerging biology of gut-brain communication. Nat Rev Neurosci, 2011; 12(8): 453-466

McEwen BS, Seeman TE. Protective and damaging effects of mediators of stress: elaborating and testing the concepts of allostasis and allostatic load. Ann N Y Acad Sci, 1999; 896: 30-47

Morris SB. Estimating effect sizes from pretest-posttest-control group designs. Org Res Methods, 2008; 11(2): 364-386

Mückschel M, Stock AK, Beste C. Psychophysiological mechanisms of interindividual differences in goal activation modes during action cascading. Cereb Cortex, 2014; 24(8): 2120-2129

Paul M, Garg K. The effect of heart rate variability biofeedback on performance psychology of basketball players. Appl Psychophysiol Biofeedback, 2012; 37(2): 131-144

Paul M, Garg K, Sandhu, JS. Role of biofeedback in optimizing psychomotor performance. Asian J Sport Med, 2012; 3(1): 29-40

Porges SW. The polyvagal perspective. Biol Psychol, 2007; 74(2): 116-143

Porges SW. The polyvagal theory: new insights into adaptvie reactions of the autonomic nervous system. Cleve Clin J Med, 2009; 76(Suppl 2): S86-S90

Pusenjak N, Grad A, Tusak M, Leskovsek M, Schwarzlin R. Can biofeedback training on psychophysiological responses enhance athletes' sports performance? a practitioner's perspective. Phys Sportsmed, 2015; 43(3): 287-289 
Prinsloo GE, Rauch HG, Derman, WE.A brief review and clinical application of heart rate variability biofeedback in sports, exercise, and rehabilitation medicine. Phys Sportsmed, 2014; 42(2): 88-99

Raymond J, Sajid I, Parkinson LA, Gruzelier JH. Biofeedback and dance performance: a preliminary investigation. Appl Psychopysiol Biofeedback, 2005; 30(1): 64-73

Rusciano A, Corradini G, Stoianov I. Neuroplus biofeedback improves attention, resilience and injury prevention in elite soccer players. Psychophysiology, 2017; 54(6): 916-926

Rusticus SA, Lovato CY. Impact of sample size and variability on the power and type 1 error rates of equivalence tests: a simulation study. Prac Assess Res Eval, 2014; 19(11): 1-10

Sellaro R, van Leusden JW, Tona KD, Verkuil, B, Nieuwenhuis S, Colzato LS. Transcutaneous vagus nerve stimulation enhances post-error slowing. J Cogn Neurosci, 2015; 27(11): 2126-2132

Steenbergen L, Sellaro R, Stock AK, Verkuli B, Beste C, Colzato LS. Transcutaneous vagus nerve (tVNS) stimulation enhances response selection during action cascading processes. Eur Neuropsychophamarcol, 2015; 25(6): 773-778

Thayer JF, Lane RD. Claude Bernard and the heart-brain connection: further elaboration of a model of neurvisceral integration. Neurosci Biobehav Rev, 2009; 33(2):81-88

van de Water T, Huijgen B, Faber I, Elferink-Gemser M. Assessing cognitive performance in badminton players: a reproducibility and validity study. J Hum Kinet, 2017; 55(1): 149-59

Vaschillo EG, Vaschillo B, Lehrer PM. Characteristics of resonance in heart rate variability stimulated by biofeedback. Appl Psychophysiol Biofeedback, 2006; 31(2): 129-142

Vaschillo EG, Vysochin YV, Rishe N. RSA biofeedback as an effective relaxation method. Appl Psychophysiol Biofeedback, 1998; 23: 136-137

Verbugh L, Scherder EJ, van Lange PA, Oosterlaan J. The key succes in elite athletes? explicit and implicit motor learning in youth elite and non-elite soccer players. J Sports Sci, 2016; 34(18):1782-1790

Wilson VE, Peper E, Gibney KH. Using the "Aha" experience with biofeedback: Enhancing body-mind integration. Biofeedback, 2004; 32: 21-25

Yildiz A, Quetscher C, Dharmadhikari S, Chmielewski W, Glaubitz B, Schmidt-Wilcke T, Beste C. Feeling safe in the plane: neural mechanisms underlying superior action control in airplane pilot trainees $-\mathrm{a}$ combined EEG/MRS study. Hum Brain Mapp,2014; 35(10): 5040-5051

Zhu Q, Tong Y, Wu T, Li J, Tong N. Comparison of the hypoglycemic effect of acarbose monotherapy in patients with type 2 diabetes mellitus consuming an Eastern or Western diet: a systematic metaanalysis. Clin Ther, 2013; 35(6): 880-99

\section{Corresponding author:}

\section{Jeffrey Cayaban Pagaduan}

College of Health and Medicine, School of Health Sciences, University of Tasmania

Newnham Drive, Newnham, Tasmania, 7248

E-mail: jeffrey.pagaduan@utas.edu.au 